\title{
Alterações funcionais e estruturais do complexo craniofacial na síndrome branquio-óculo-facial: relato de caso
}

\author{
Functional and structural alterations of the \\ craniofacial complex in the branchio-oculo- \\ facial syndrome: case report
}

\section{Alteraciones funcionales y estructurales del complejo craneofacial en el síndrome branquio-óculo-facial: relato de caso}

\author{
Gabriela Ribeiro Schilling* \\ Gustavo Jungblut Kniphoff* \\ Mariana Barboza da Silva* \\ Lisiane De Rosa Barbosa* \\ Márcia Salgado Machado* \\ Maria Cristina de Almeida Freitas Cardoso* \\ Marcia Angelica Peter Maahs*
}

\section{Resumo}

Introdução: A Síndrome Branquio-Óculo-Facial é uma doença autossômica rara com expressão variável, dependente das mutações genéticas, cujo fenótipo caracteriza-se por alterações oculares, auriculares e craniofaciais. Objetivo: Descrever características clínicas, alterações funcionais e estruturais do complexo craniofacial de sujeito com síndrome branquio-óculo-facial. Método: Paciente de 13 anos

*Universidade Federal de Ciências da Saúde Porto Alegre (UFCSPA) - Porto Alegre, Rio Grande do Sul, RS, Brasil

Contribuição dos autores:

GRS: concepção do relato de caso, coleta e interpretação de dados e escrita do artigo; GJK e MBS: coleta de dados e escrita do artigo; LRB e MSM: escrita do artigo e revisão crítica do conteúdo; MCAFC: interpretação de dados, escrita do artigo e revisão crítica do conteúdo; MAPM: concepção do relato de caso, coleta e interpretação de dados, escrita do artigo e revisão crítica do conteúdo.

E-mail para correspondência: Gabriela Ribeiro Schilling - gabrielar.schilling@gmail.com Recebido: 02/01/2019

Aprovado: 02/08/2019 
e 3 meses, respiradora oral com perda auditiva condutiva de grau moderadamente severo em ambas as orelhas, diagnosticada com síndrome branquio-óculo-facial, apresentou: fissura labiopalatina transforame bilateral completa corrigida por labioplastia e palatoplastia primárias, assimetria facial, fístula em região anterior de palato duro, atresia maxilar transversa, dentinogênese imperfeita, trespasse horizontal negativo, oclusão Classe I de Angle e mordida aberta anterior e lateral bilateralmente, desvio severo da linha média superior para a esquerda, incisivo lateral superior permanente semi-erupcionado por vestibular do canino superior decíduo do lado esquerdo, retenção prolongada do segundo molar inferior decíduo direito, apinhamento dentário inferior, hipotonia e posicionamento inadequado de língua, ausência de vedamento labial em repouso, deglutição adaptada, alteração na mobilidade de lábios, bochechas e palato mole com escape de ar nasal na fala, caracterizando disfunção velofaríngea. Sujeitos com fissura lábiopalatina podem apresentar grande variedade de alterações na produção dos fones. Paciente apresenta crescimento deficiente da maxila que, como relatado na literatura, pode alterar o desenvolvimento do terço médio da face com repercussão na oclusão dentária, fala e formato do nariz. Conclusão: As alterações clínicas funcionais e estruturais relatadas são na maioria do complexo craniofacial, demonstrando a importância da otorrinolaringologia, fonoaudiologia e odontologia na terapêutica interdisciplinar dos pacientes com a síndrome.

Palavras-chave: Síndrome Brânquio-Oculo-Facial; Fonoaudiologia; Ortodontia; Fala; Dente

\section{Abstract}

Introduction: Branchio-Oculo-Facial Syndrome (BOFS) is a rare autosomal disease with variable expression, dependent on genetic mutations, whose phenotype is characterized by ocular, hearing and craniofacial alterations. Purpose: describe the clinical features, the functional and structural alterations in the craniofacial complex of a subject with branchio-oculo-facial syndrome. Method: A 13-year and 3-month-old girl, with moderately severe conductive bilateral hearing loss diagnosed with BOFS, presented: bilateral cleft lip and palate treated by labioplasty and palatoplasty, facial asymmetry, anterior maxillary fistula, transverse maxillary atresia, imperfect dentinogenesis, negative horizontal trespass, Angle Class I bilateral, anterior and lateral open bite on both sides, severe left superior midline deviation, eruption by vestibular of the superior canine on the left side, prolonged retention of the second inferior molar right deciduous, lower dental crowding, hypotonia and inadequate tongue positioning, absence of labial resting at rest, adapted swallowing, alteration in mobility of lips, cheeks and palate with nasal air exhaust in speech, characterizing velopharyngeal dysfunction. There are few publications of BOFS, given its rarity. Subjects with cleft lip and palate may present a wide variety of changes in the production of headphones. Patient presents deficient growth of the maxilla which, as reported in the literature, may alter the development of the middle third of the face with repercussion in dental occlusion, speech and nose shape. Conclusion:_The functional and structural clinical alterations reported are the majority of the craniofacial complex, demonstrating the importance of otorhinolaryngology, speech therapy and orthodontics in the interdisciplinary therapy of patients with BOFS.

Keywords: Branchio-Oto-Facial Syndrome; Speech, Language and Hearing Sciences; Orthodontics; Speech; Tooth.

\section{Resumen}

Introducción: El síndrome branquio-oculo-facial (BOFS) es una enfermedad autosómica rara con expresión variable, dependiente de las mutaciones genéticas, caracterizada por alteraciones oculares, auriculares y craneofaciales. Propósito: describir características clínicas, alteraciones funcionales y estructurales del complejo craneofacial de un sujeto con BOFS. Método: Niña de 13 años y 3 meses de edad, con pérdida de audición conductiva moderadamente grave bilateralmente diagnosticada con SBOF, presentó: paladar y labio hendido bilateral tratado por labioplastia y palatoplastia primarias, asimetría facial, fístula maxilar anterior, atresia maxilar transversal, dentinogénesis imperfecta, traspaso horizontal negativo, clase I de Angle bilateral, mordida abierta anterior y lateral bilateralmente, desviación severa de la línea media superior izquierda, erupción vestibular del canino superior del lado izquierdo, retención 
prolongada del segundo molar inferior derecho deciduo, apiñamiento dental, hipotonía e inadecuada colocación de la lengua, ausencia de sello labial en reposo, deglución adaptada, alteración de movilidad de labios, mejillas y velo del paladar con escape de aire nasal y disfunción velofaríngea Hay pocas publicaciones de BOFS, dada su rareza. Los sujetos con labio y paladar hendido pueden presentar una gran variedad de cambios en la producción de auriculares. El paciente presenta crecimiento deficiente del maxilar que, según se informa en la literatura, puede alterar el desarrollo del tercio medio de la cara con repercusión en la oclusión dental, habla y la forma de la nariz. Conclusión: Alteraciones clínicas funcionales y estructurales la mayoría del complejo craniofacial. Eso demuestra la importancia de otorrinolaringología, fonoaudiología y odontología en la terapia interdisciplinaria de pacientes con SBOF.

Palabras clave: Síndrome Brânquio-Otorrenal; Fonoaudiología; Ortodoncia; Habla; Diente

\section{Introdução}

A síndrome branquio-óculo-facial (SBOF) é uma doença autossômica rara com expressão altamente variável e dependente das mutações genéticas presentes ${ }^{1}$, sendo descritos 81 casos na literatura ${ }^{2}$. O gene TFAP2A, localizado no cromossomo 6, pode apresentar-se deletado ou mutado nos portadores de $\mathrm{SBOF}^{3}$. O fenótipo da síndrome se caracteriza por alterações oculares, auriculares e craniofaciais ${ }^{1}$.

As alterações craniofaciais frequentemente encontradas nos portadores de SBOF são: pseudofissuras labiais, fissuras labiais associadas ou não a fissuras de palato ${ }^{4}$, palato estreito e ogival, nariz assimétrico e malformado com ponte larga e ponta achatada $^{4,5}$. Os defeitos branquiais cervicais pós-auricular bilaterais com lesões hemangiomatosas cobertas por uma porção de pele anormal, associados ou não a fossetas pré-auriculares ou auriculares, perda auditiva e posicionamento baixo das orelhas também são característicos da síndrome ${ }^{4,5}$.

Alterações oculares como microftalmia, coloboma, catarata, obstrução do ducto nasolacrimal, miopia, estrabismo e ptose são encontradas nos sujeitos com $\mathrm{SBOF}^{4,5}$. Malformações extra craniofaciais são raras nesta síndrome, sendo as anormalidades renais as mais frequentes ${ }^{6}$. O sujeitos com SBOF podem apresentar, ocasionalmente, embranquecimento prematuro dos cabelos e cistos no couro cabeludo 5 . A SBOF pode estar associada a retardo mental moderado ${ }^{5}$ e perda auditiva em $70 \%$ dos sujeitos ${ }^{7}$. A ausência de incisivos superiores decíduos foi relatada como um sinal presente na $\mathrm{SBOF}^{8}$

Devido à raridade de estudos relacionados à SBOF, justifica-se a descrição deste relato de caso que objetiva apresentar as características clínicas presentes, em especial as inúmeras alterações funcionais e estruturais do complexo craniofacial.

\section{Relato de caso}

A paciente deste estudo frequenta o ambulatório do Sistema Único de Saúde no Hospital da Criança Santo Antônio (pertencente ao Complexo Hospitalar Santa Casa de Misericórdia de Porto Alegre/RS - Brasil). Neste ambulatório são desenvolvidas atividades do "Projeto de Extensão Fissuras Lábio-palatinas" da Universidade Federal de Ciências da Saúde de Porto Alegre (UFCSPA), que visa a atenção interdisciplinar desde o nascimento até os dezoito anos. As atividades de pesquisa realizadas no mesmo foram aprovadas pelo Comitê de Ética em Pesquisa da instituição de saúde proponente, sob o número1.900.382, sendo vinculado ao projeto de pesquisa "Ações Clínicas em fonoaudiologia de origem musculoesquelética". Os responsáveis pela paciente assinaram o Termo de Consentimento Livre Esclarecido e o Termo para Uso de Imagens, assim como a paciente assinou o Termo de Assentimento do Menor, todos autorizando a utilização dos dados e das imagens presentes neste estudo. Na avaliação fonoaudiológica estrutural e funcional, bem, como, na avaliação odontológica clínica foram utilizados protocolos próprios, desenvolvidos pelo grupo de pesquisa do projeto, para esta população.

Paciente com SBOF, 13 anos e 3 meses, branca, sexo feminino, primeira filha de pais hígidos e não-consanguíneos, realizou avaliação fonoaudiológica e odontológica no ambulatório do "Projeto de Extensão Fissuras Lábio-palatinas", após inúmeras avaliações e acompanhamento médico ao longo dos anos, incluindo a otorrinolaringologia. A menina realiza terapia fonoaudiológica neste ambulatório. 
Sua história familiar é negativa para quaisquer defeitos congênitos ou doenças genéticas.

O diagnóstico clínico de SBOF foi estabelecido pela equipe de genética clínica do hospital. A paciente foi encaminhada à equipe de genética médica de outro hospital de referência para a realização de avaliações e exames complementares de doenças genéticas, como os testes moleculares que determinam o gene alterado.

Como características clínicas da síndrome na região craniofacial, a menina apresentava fissura labiopalatina bilateral completa, corrigida por labioplastia aos 2 meses e palatoplastia aos 12 meses; coloboma da íris e de nervo óptico; obstrução do ducto nasolacrimal; perda auditiva condutiva de grau moderadamente severo em ambas as orelhas; orelhas com implantação baixa (Figura 2); nariz assimétrico com ponte larga e ponta achatada (Figura 1) e orifícios em região branquial (retroauricular) bilateralmente. Como dados complementares de saúde, podemos citar rim direito multicístico, retardo no desenvolvimento neuropsicológico, escoliose tóraco-lombar de baixo valor angular, epilepsia, blefarofimose, deficiência visual e estenose do canal auditivo direito.

À avaliação fonoaudiológica de motricidade orofacial, a paciente apresentou as seguintes caraterísticas orofaciais: presença de fístula em região anterior de palato duro, apresentando-se este atrésico, hipodesenvolvimento de maxila, alteração na mobilidade de lábios (com hipomovimentação de lábio superior) e bochechas, hipotonia e posicionamento inadequado de língua (posicionada no assoalho oral e com interposição lingual), assimetria facial (Figura 1), ausência de vedamento labial em repouso (Figuras 1 e 2) e alteração da mobilidade de palato mole com escape de ar nasal na fala, caracterizando disfunção velofaríngea.

As funções estomatognáticas de deglutição e mastigação também foram avaliadas. À avaliação de deglutição, a paciente apresentou múltiplas deglutições para sólidos e líquidos e hiperativação do músculo mentual durante a execução da função. Na mastigação de sólidos, a paciente abocanhava pequenas quantidades de alimento com lentidão no trânsito oral. Tais achados caracterizam um padrão de deglutição adaptado às condições anatômicas presentes, inclusive ao uso do aparelho ortodôntico na arcada superior.

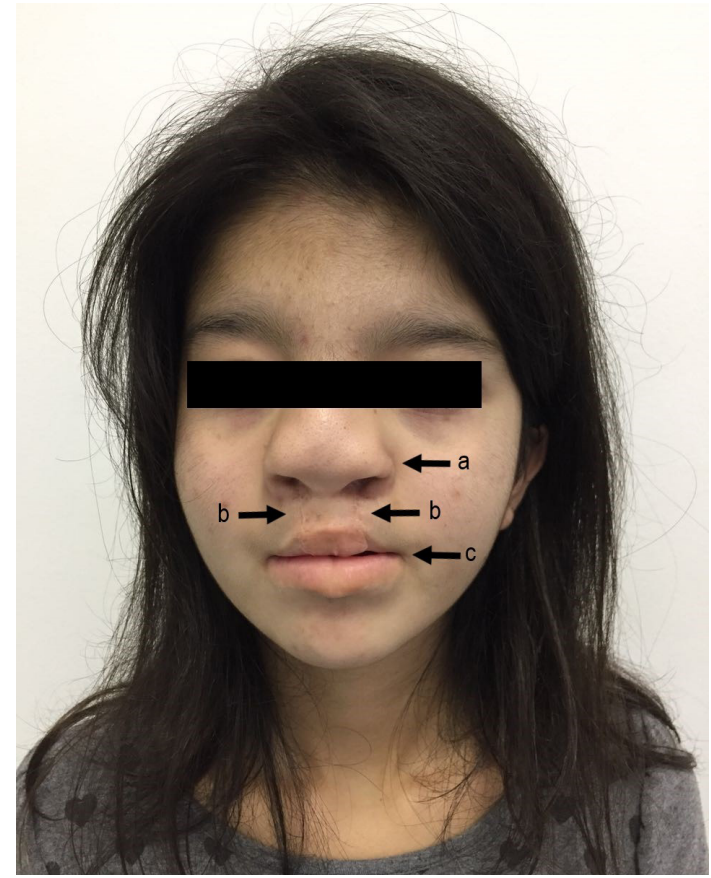

Figura 1. Fotografia frontal da face. Destaque para assimetria facial, nariz assimétrico com ponte larga e ponta achatada (a), cicatriz na região da labioplastia bilateral (b) e ausência de vedamento labial em repouso (c).

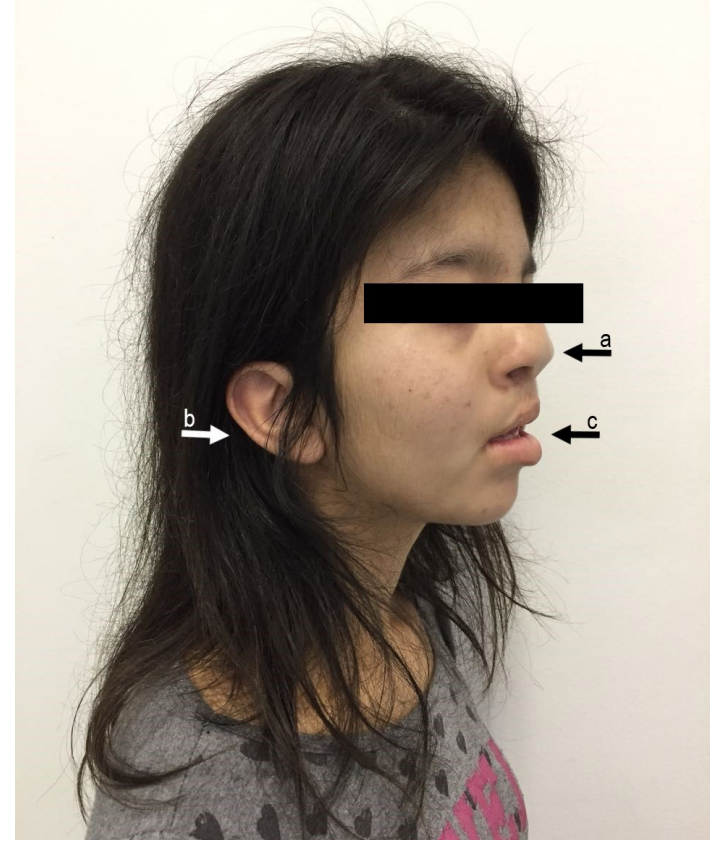

Figura 2. Fotografia de perfil do lado direito da face. Destaque para nariz com ponta achatada (a), implantação baixa da orelha (b) e ausência de vedamento labial em repouso (c). 
À avaliação de fala foram encontradas alterações fonológicas e fonéticas. Apresentou escape de ar nasal nos fones fricativos, com interposição lingual na produção dos fones anteriores; dessonorização de plosivas e fricativas; substituição dos fonemas palatais pelos linguodentais; substituição da líquida alveolar em coda pelo fonema velar vibrante; produção de plosivas dorso-médio-palatais e imprecisão na produção da líquida alveolar em onset inicial e alteração do ponto articulatório do fone /1/, elevando o ápice da língua à face vestibular dos incisivos superiores na produção do mesmo.

Em relação às características clínicas ortodônticas apresentadas pela paciente, após início do tratamento ortodôntico interceptativo, destacaram-se: Classe I de Angle (Figuras 4 e 5), mordida aberta anterior (Figura 3) e lateral do lado direito e esquerdo (Figuras 4 e 5), desvio severo da linha média superior para esquerda (Figura 3), incisivo lateral superior permanente semi-erupcionado por vestibular do canino superior decíduo do lado esquerdo (Figura 5), retenção prolongada do segundo molar decíduo inferior do lado direito (Figura 4) e apinhamento dentário inferior (Figuras 3, 4 e 5). Ademais, apresenta diagnóstico odontológico de dentinogênese imperfeita e sinais de gengivite (Figura 3).

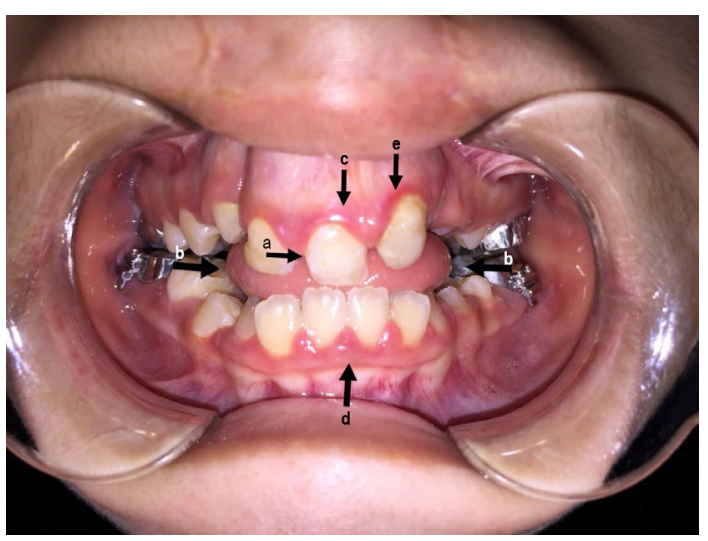

Figura 3. Fotografia intra-oral em oclusão de frente. Destaque para dentinogênese imperfeita visível nos incisivos superiores (a), mordida aberta anterior e bilateral e interposição lingual anterior e bilateral (b), desvio severo da linha média superior para o lado esquerdo (c), apinhamento dentário inferior (d) e sinais de gengivite (e).

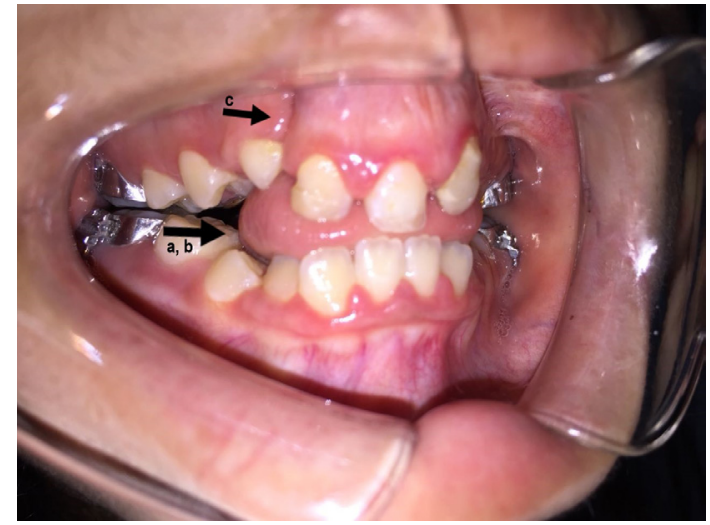

Figura 4. Fotografia em oclusão do lado direito. Destaque para mordida aberta lateral (a), interposição lingual (b) e presença da fissura alveolar do lado direito (c).

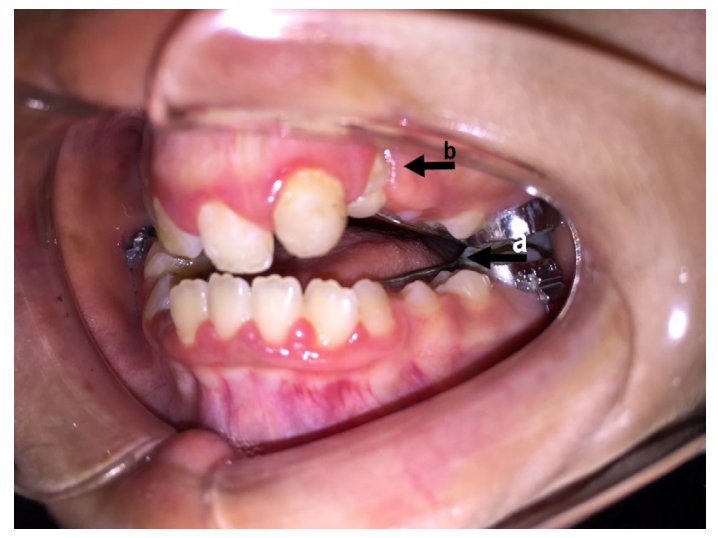

Figura 5. Fotografia em oclusão do lado esquerdo. Destaque para mordida aberta lateral do lado esquerdo (a) e incisivo lateral permanente superior semi-erupcionado por vestibular do canino superior decíduo do lado esquerdo no local da fissura alveolar (b).

A paciente realiza tratamento ortodôntico interceptativo fora dos âmbitos do projeto de extensão, por meio do uso de disjuntor maxilar e arco lingual. A terapia fonoaudiológica desenvolvida até o momento visa adequar as questões relacionadas à fala, disfunção velofaríngea e alterações orofaciais relatadas. A paciente permanecerá em atendimento até conclusão dos respectivos planos terapêuticos ortodônticos e fonoaudiológicos.

\section{Discussão}

Na literatura há escassez de publicações relacionadas à $\mathrm{SBOF}$, visto a raridade da mesma. 
Em uma revisão de literatura realizada em 2010 foram encontrados apenas 81 artigos relacionados ao tema ${ }^{2}$. Relatos que descrevam as características fonoaudiológicas e odontológicas dos pacientes com SBOF são ainda mais $\operatorname{raros}^{8}$.

A paciente deste relato apresentou aspectos clínicos relacionados à SBOF já descritos na literatura como alterações auditivas, craniofaciais e oculares $^{4,5}$. A perda auditiva, que ocorre em $70 \%$ dos $\operatorname{casos}^{7}$, e pode ter repercussões na fala, como o processo fonológico de dessonorização de plosivas e fricativas observado em fala espontânea e dirigida da paciente. Podem ocorrer malformações extracraniofaciais raras na SBOF, como anormalidades renais ${ }^{6}$; a paciente deste estudo apresentou rim direito multicístico e retardo mental ${ }^{5}$, exibindo retardo no desenvolvimento neuropsicológico.

O estudo de Flores $2015^{8}$ relata alteração de fala de paciente com SBOF, entretanto não traz detalhamentos sobre os tipos de substituições ou processos adaptativos de fala realizados pelo sujeito ${ }^{8}$. Portadores de SBOF, com fissura labiopalatina, podem apresentar alterações de fala relacionadas a alterações dento-oclusais e a disfunção velofaríngea devido às anomalias anatomofuncionais do palato9. Sujeitos com FLP podem apresentar uma grande variedade de alterações no ponto e no modo de produção dos fones ${ }^{9}$, como verificado junto à paciente deste estudo. A articulação apresentada pela paciente na produção dos fonemas líquidos laterais, utilizando a face vestibular dos incisivos superiores em contato com o ápice de língua, pode ser justificada pela presença de imperfeições estruturais marcantes que culminam em articulações improváveis de serem realizadas ${ }^{9}$, como o trespasse horizontal negativo, mordida aberta anterior e bilateral e atresia maxilar transversa. Embora as avaliações fonoaudiológica e odontológica tenham sido realizadas durante tratamento ortodôntico, a presença do disjuntor maxilar indica que havia atresia maxilar transversa; e o arco lingual é mantedor de espaço para as trocas dentárias, não interferindo nas alterações encontradas. O posicionamento de língua em repouso e durante as funções estomatognáticas pode ter sido influenciado pela presença dos aparatos ortodônticos, porém, as alterações de motricidade orofacial encontradas normalmente estão associadas às alterações estruturais presentes.

A deficiência ânteroposterior de maxila pode aparecer em pacientes submetidos a cirurgias como a labioplastia e palatoplastia ${ }^{10}$, cirurgias estas rea- lizadas pela paciente relatada neste estudo, o que pode ter gerado o trespasse horizontal negativo. O crescimento deficitário da maxila pode alterar o desenvolvimento do terço médio da face com posterior repercussão na oclusão dentária, na fala e na forma do nariz ${ }^{10}$, como no presente caso.

\section{Conclusão}

Algumas alterações funcionais e estruturais do complexo craniofacial presentes nesta paciente com SBOF estão de acordo com a literatura, já outras ainda não haviam sido relatadas. Embora exista heterogeneidade do fenótipo, são necessários novos estudos de pesquisa com amostras significativas para caracterizar as alterações encontradas como características da síndrome. Fica o alerta da necessidade de intervenção interdisciplinar na SBOF o mais precocemente possível, destacando a importância da otorrinolaringologia, fonoaudiologia e odontologia devido às alterações encontradas.

\section{Referências}

1. Galliani E, Burglen L, Kadlub N, Just W, Sznajer Y, de Villemeur TB,et al. Craniofacial phenotype in the branchiooculo-facial syndrome: four case reports. Cleft Palate Craniofac J. 2012; 49(3): 357-64.

2. Al-Dosari MS, Almazyad M, Al-Ebdi L, Mohamed JY, Al-Dahmash S, Al-Dhibi H, et al. Ocular manifestations of branchio-oculo-facial syndrome: report of a novel mutation and review of the literature. Mol Vis. 2010; 16: 813-18.

3. Milunsky JM, Maher TA, Zhao G, Roberts AE, Stalker HJ, Zori RT, et al. TFAP2A mutations result in branchio-oculo-facial syndrome. Am J Hum Genet. 2008; 82(5): 1171-7.

4. Fujimoto A, Lipson M, Lacro RV, Shinno NW, Boelter W, Jones KL, et al. New autosomal dominant branchio-oculo-facial syndrome. Am J Med Genet. 1987; 27(4): 943-51.

5. Lin AE, Gorlin RJ, Lurie IW, Brunner HG, van der Burgt I, Naumchik IV, et al. Further delineation of the branchio-oculofacial syndrome. Am J Med Genet. 1995; 56(1): 42-59.

6. Lin AE, Doherty R, Lea D. Branchio-oculo-facial and branchio-oto-renal syndromes are distinct entities. Clin Genet 1992; 41(4): 221-2.

7. Milunski JM, MAher TM, Zhao G, Wang Z, Mulliken JB, Chitayat D, et al. Genotype-phenotype analysis os the Branchio-Oculo-Facial Syndrome. Am J Med Genet A. 2011; 155A(1): 22-32.

8. Flores JBG, Ocañasa CEE, Menchaca HRM, Alanísa MGT, Silva GR. New oral manifestations of Branchio-oculo-facial syndrome. Case report. Arch Argent Pediatr 2015; 113(1): 14-6.

9. Hanayama EM. Distúrbios de comunicação nos pacientes com sequela de fissura labiopalatina. Rev Bras Cir Craniomaxilofac 2009; 12(3): 118-24.

10. Ribeiro EM, Moreira ASCG. Atualização sobre o tratamento multidisciplinar das fissuras labiais e palatinas. RBPS 2005; 18 (1): 31-40. 